\title{
Rotation period and magnetic field morphology of the white dwarf WD 0009+501
}

\author{
G. Valyavin ${ }^{1,2}$, S. Bagnulo ${ }^{3}$, D. Monin ${ }^{4}$, S. Fabrika ${ }^{2}$, B.-C. Lee ${ }^{1}$, G. Galazutdinov ${ }^{1,2}$, G. A. Wade ${ }^{5}$, and T. Burlakova ${ }^{2}$ \\ ${ }^{1}$ Korea Astronomy and Space Science institute, 61-1, Whaam-Dong, Youseong-Gu, Taejeon 305-348, Republic of Korea \\ e-mail: gendoz@boao.re.kr \\ 2 Special Astrophysical Observatory, Russian Academy of Sciences, Nizhnii Arkhyz, Karachai Cherkess Republic \\ 357147, Russia \\ 3 European Southern Observatory, Alonso de Cordova 3107, Santiago, Chile \\ ${ }^{4}$ Département de Physique et d'Astronomie, Université de Moncton, Moncton, NB, E1A 3E9, Canada \\ 5 Department of Physics, Royal Military College of Canada, PO Box 17000 Stn "FORCES", Kingston, Ontario, \\ K7K 7B4, Canada
}

Received 5 January 2005 / Accepted 8 May 2005

\begin{abstract}
We present new spectropolarimetric observations of the weak-field magnetic white dwarf WD 0009+501. From these data we estimate that the star's longitudinal magnetic field varies with the rotation phase from about $-120 \mathrm{kG}$ to about $+50 \mathrm{kG}$, and that the surface magnetic field varies from about $150 \mathrm{kG}$ to about $300 \mathrm{kG}$. Earlier estimates of the stellar rotation period are revised anew, and we find that the most probable period is about $8 \mathrm{~h}$. We have attempted to recover the star's magnetic morphology by modelling the available magnetic observables, assuming that the field is described by the superposition of a dipole and a quadrupole. According to the best-fit model, the inclination of the rotation axis with respect to the line of sight is $i=60^{\circ} \pm 20^{\circ}$, and the angle between the rotation axis and the dipolar axis is $\beta=111^{\circ} \pm 17^{\circ}$. The dipole strength at the pole is about $340 \mathrm{kG}$, and the quadrupolar strength is about $300 \mathrm{kG}$.
\end{abstract}

Key words. stars: white dwarfs - stars: magnetic fields - stars: rotation - stars: individual: WD 0009+501

\section{Introduction}

It is generally assumed that magnetic white dwarfs are the descendants of magnetic Ap/Bp stars. This assumption, if true, suggests that magnetic white dwarfs, as relics of stellar cores, are unique indicators revealing remnants of internal fields of intermediate-mass stars. Therefore, spectropolarimetric investigation of magnetic white dwarfs is of fundamental importance for understanding the origin and evolution of global stellar magnetic fields. Knowing white dwarf magnetic field geometries, strengths and rotation periods, one may restrict assumptions in theories of the evolution of intermediate mass stars. For instance, the rotation periods and surface magnetic fields of white dwarfs could be used to find the angular momentum and magnetic flux lost by a star during formation of the degenerate core and associated mass loss. The distribution of white dwarf magnetic field strengths could yield information about whether magnetic white dwarfs represent an independent class of strongly-magnetic degerate stars, or if they are those objects with the strongest fields in a continuous distribution of field strengths (Angel et al. 1981; Wickramasinghe \& Ferrario 2000, 2005).
While the theory of white dwarf magnetic fields has been developing quite successfully (for instance Wendell et al. 1987; Muslimov et al. 1995), observational data are still required, especially in a low-field regime (Schmidt \& Smith 1994, 1995; Valyavin et al. 2003; Liebert et al. 2003). Presently, only a few white dwarfs with the fields below $1 \mathrm{MG}$ are known (Aznar Cuadrado et al. 2004, and references therein). Their rotation and magnetic field geometries are poorly studied, although some progresses have been made recently with the work by Maxted et al. (2000) on WD 1953-011, a white dwarf characterised by one of the weakest measured fields. Maxted et al. (2000) discovered that the magnetic morphology of WD 1953-011 is dominated by a strong non-dipolar component ( $\sim 500 \mathrm{kG}$; see also Wade et al. 2004). This result has shown that the interpretation of the spectra of sub-megaGauss magnetic white dwarfs which is a crucial point for understading the processes of stellar magnetic field evolution. In order to continue the discussion, here we present a study of the rotation period and the magnetic morphology of another weak-field white dwarf: WD 0009+501.

WD 0009+501 (=GJ 1004) was first discovered as a low field magnetic degenerate by Schmidt \& Smith (1994). In twelve observations obtained during four observing nights, 
they found variations of the longitudinal magnetic field over the range $B_{1}=-100 \div+9 \mathrm{kG}$ with error bars $\sigma\left(B_{1}\right)=8 \div 15 \mathrm{kG}$. Assuming the magnetic field varies because of the star's rotation, they found the probable period to be between 2 and $20 \mathrm{~h}$. Power spectrum analysis displayed various periods in this region with some preference for shorter periods. No variation of the magnetic field was found in a pair of spectra separated by $12 \mathrm{~min}$, but significant difference was detected in observations separated by $5 \mathrm{~h}$. Field variation of about $100 \mathrm{kG}$ was detected.

More recently (Fabrika et al. 2003) we repeated Zeeman observations of this white dwarf. The series of observations consisted of 21 consecutive Zeeman spectra taken with 5- and 10-min exposures. These measurements confirmed the presence of a varying magnetic field in WD $0009+501$. The rotation period was estimated about $2 \mathrm{~h}$.

To further study the magnetic field configuration we obtained additional observations at the $6 \mathrm{~m}$ Russian telescope BTA, and performed new determinations of the mean longitudinal field, mean field modulus and mean asymmetry of the longitudinal field multiplied by $v_{\mathrm{e}} \sin i$ (crossover). In fact, we found that our new observations are not consistent with the earlier estimate of the rotation period. In this paper we present a new determination of the rotation period and a magnetic model consistent with all determinations of the magnetic observables.

\section{New observations and data reduction}

The observations were carried out in the course of six nights from December 29, 2003 to January 3, 2004 with the prime focus spectrograph-polarimeter UAGS of the $6 \mathrm{~m}$ telescope $(R \sim 2000)$. The instrument and observational technique are described in detail by Afanasiev et al. (1995) and by Naydenov et al. (2002). The spectropolarimeter is a standard spectrograph equipped with a rotatable quarter-wave plate and a beamsplitting analyzer. Each CCD image contains two spectra of opposite circular polarizations split by the analyzer. A circular polarization observation consists of a series of paired exposures obtained at two orthogonal orientations of the waveplate (the modulation technique, similar to that described by Schmidt \& Smith 1994). We estimated a priori that the time-scale for the variability of the observed magnetic field could be as short as a few minutes. For this reason, but also to limit the parasitic influence of atmospheric sincillation, we decided to set the integration time of each exposure to $150 \mathrm{~s}$.

The reduction of the CCD images were carried out using the image processing packages MIDAS (context "LONG" for long-slit spectrographs). The applied procedures were mostly standard including the following steps: cosmic ray hits removing, electronic bias substraction, flat-fielding, 2D wavelength calibration, sky background substraction, spectrum extraction. Finally we obtained a series of pairs of left and right circular polarized spectra that were used to search for the rotation period as described in Sect. 3.

\section{Period determination}

For the search of the star's rotation period we used the determinations of disk-averaged longitudinal component of the magnetic field $B_{1}$ obtained from our spectra. Longitudinal field measurements were first obtained via analysis of the displacement between the positions of $\mathrm{H} \alpha$ in the spectra of opposite circular polarizations. For the determination of the line profile displacement we used a technique based on estimate of the autocorrelation function of Zeeman shifted spectral features using the discrete Fourier transform (Scargle 1989). This technique appears to be relatively insensitive to uncertainties in the continuum determination (Klochkova et al. 1996). For the determination of the mean longitudinal field we used the formula:

$\Delta \lambda=\lambda_{R}-\lambda_{L}=9.34 \times 10^{-13} g_{\mathrm{eff}} B_{1} \lambda^{2}$

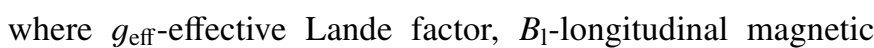
field and $\lambda$-wavelength (for more detail see Monin et al. 2002, and references therein). A preliminary inspections to the series of longitudinal field values allowed us to rule out a variability time-scale shorter than $600 \mathrm{~s}$. Hence we decided to consider a series of longitudinal field determinations where each individual value is obtained from the weighted average of four (consecutive) points. We obtained a series of field determinations where each point correponds to $600 \mathrm{~s}^{\text {exposure }}{ }^{1}$. These values are listed in Table 1, where we also present reprocessed and averaged data (JD $=2451423$ at the table) that were obtained earlier with spectrograph SP-124 of the 6-m telescope (described by Fabrika et al. 2003).

To determine the rotation period we applied the LaflerKinman method (Lafler \& Kinman 1965), as modified by Goransky (2004). In case of non-uniformly sampled and highly noisy data, this method leads to more accurate results than the traditional Fourier power spectrum analysis.

Analysis of the power spectrum of the data revealed a strong signal indicating a probable period ranging from $5 \mathrm{~h}$ to 1 day (Fig. 1). This is consistent with the period range $2<P<20$ h suggested by Schmidt \& Smith (1994). A detailed study of the periodogram showed that the most significant sinusoidal signal corresponds to about $8 \mathrm{~h}(P=8 \mathrm{~h} \pm 5 \mathrm{~min})$. Other peaks are present around 12 and $16 \mathrm{~h}$. However, the longitudinal field curves obtained using a rotation period of $12 \mathrm{~h}$ or $16 \mathrm{~h}$ show a bimodal structure that cannot be interpreted in terms of a simple low-order multipolar expansion of the magnetic field. To test the reliability of the $8 \mathrm{~h}$ period we performed a "spectral cleaning" of the periodogram as described by Terebizh (1992). We fit and extracted a sinusoidal function of a $8 \mathrm{~h}$ period from the observed data, and carried out the periodogram analysis for the residual data. If the tested period is correct, we expect the residual periodogram be nearly "white noise" structure (Terebizh 1992). If real signal has multi-frequency nature, the spectral cleaning needs to be carried out iteratively using multifrequency trial harmonic functions. Our analysis indicates that the best result is obtained with the $8 \mathrm{~h}$ period (the lower panel in Fig. 1).

1 With two exceptions: because of weather conditions, in two different cases we had to co-add spectra for a total of 25 and $30 \mathrm{~m}$, respectively; see Table 1. 
Table 1. Determinations of mean longitudinal field obtained as explained in Sect. 2. Column 1 is the Julian Date, Col. 2 is the equivalent exposure time, Col. 3 is the mean longitudinal magnetic field (in kG), Col. 4 is the associated error bar (in kG).

\begin{tabular}{|c|c|c|c|c|c|c|c|}
\hline $\mathrm{JD}-2400000$ & $\operatorname{Exp}(\mathrm{s})$ & $B_{1}(\mathrm{kG})$ & $\sigma(\mathrm{kG})$ & $\mathrm{JD}-2400000$ & $\operatorname{Exp}(\mathrm{s})$ & $B_{1}(\mathrm{kG})$ & $\sigma(\mathrm{kG})$ \\
\hline 51423.462 & 1500 & -67 & 10 & 53004.15871 & 600 & 52 & 18 \\
\hline 51423.478 & 1200 & -98 & 13 & 53004.16712 & 600 & 35 & 14 \\
\hline 51423.493 & 1200 & -105 & 12 & 53004.17582 & 600 & 54 & 12 \\
\hline 51423.508 & 1200 & -122 & 12 & 53004.24442 & 600 & 0 & 13 \\
\hline 51423.523 & 1200 & -116 & 15 & 53004.25570 & 600 & -1 & 14 \\
\hline 51423.531 & 900 & -102 & 16 & 53004.26512 & 600 & -8 & 21 \\
\hline 51423.539 & 600 & -108 & 15 & 53005.16007 & 1500 & 61 & 27 \\
\hline 51423.547 & 600 & -112 & 13 & 53006.14914 & 600 & 24 & 13 \\
\hline 51423.554 & 600 & -110 & 13 & 53006.15736 & 600 & 49 & 13 \\
\hline 51423.562 & 600 & -101 & 12 & 53006.16564 & 600 & 48 & 11 \\
\hline 53003.15306 & 600 & 84 & 10 & 53006.17401 & 600 & 60 & 11 \\
\hline 53003.16255 & 600 & 45 & 13 & 53006.18243 & 600 & 54 & 10 \\
\hline 53003.17105 & 600 & 57 & 18 & 53006.19954 & 600 & 65 & 10 \\
\hline 53003.17976 & 600 & 34 & 15 & 53006.21667 & 600 & 54 & 10 \\
\hline 53003.18935 & 600 & 26 & 18 & 53006.22521 & 600 & 37 & 11 \\
\hline 53003.19806 & 600 & 47 & 13 & 53006.23373 & 600 & 20 & 12 \\
\hline 53003.20656 & 600 & 50 & 16 & 53006.24227 & 600 & 35 & 12 \\
\hline 53003.21513 & 600 & 25 & 14 & 53006.25063 & 600 & 8 & 10 \\
\hline 53003.22362 & 600 & 29 & 18 & 53006.25910 & 600 & 4 & 12 \\
\hline 53003.23238 & 600 & 58 & 20 & 53006.26747 & 600 & -11 & 10 \\
\hline 53003.24116 & 600 & 15 & 18 & 53006.27362 & 600 & -30 & 10 \\
\hline 53003.24972 & 600 & 22 & 17 & 53006.28170 & 600 & -59 & 10 \\
\hline 53003.25831 & 600 & -27 & 18 & 53006.29233 & 600 & -56 & 11 \\
\hline 53003.26672 & 600 & -32 & 16 & 53006.30086 & 600 & -89 & 23 \\
\hline 53003.27531 & 600 & -51 & 15 & 53006.30889 & 600 & -69 & 12 \\
\hline 53003.28373 & 600 & -14 & 15 & 53007.14476 & 600 & 75 & 10 \\
\hline 53003.29223 & 600 & -74 & 18 & 53007.15302 & 600 & 55 & 10 \\
\hline 53003.30066 & 600 & -53 & 16 & 53007.16151 & 600 & 54 & 10 \\
\hline 53003.30921 & 600 & -113 & 16 & 53007.17095 & 600 & 60 & 10 \\
\hline 53003.31784 & 600 & -91 & 18 & 53007.18220 & 600 & 65 & 11 \\
\hline 53003.32629 & 600 & -112 & 14 & 53007.19011 & 600 & 68 & 12 \\
\hline 53003.33477 & 600 & -133 & 14 & 53007.19797 & 600 & 44 & 11 \\
\hline 53003.34319 & 600 & -124 & 14 & 53007.20591 & 600 & 65 & 10 \\
\hline 53003.35171 & 600 & -135 & 15 & 53007.21409 & 600 & 50 & 10 \\
\hline 53003.36033 & 600 & -101 & 14 & 53007.22229 & 600 & 33 & 10 \\
\hline 53003.36896 & 600 & -101 & 13 & 53007.23095 & 600 & 9 & 12 \\
\hline 53003.37737 & 600 & -108 & 15 & 53008.13248 & 600 & 39 & 13 \\
\hline 53003.38564 & 600 & -67 & 18 & 53008.14073 & 600 & 25 & 10 \\
\hline 53003.39399 & 600 & -55 & 16 & 53008.14909 & 600 & 54 & 15 \\
\hline 53003.40234 & 600 & -72 & 12 & 53008.15723 & 600 & 59 & 11 \\
\hline 53003.41075 & 600 & -55 & 15 & 53008.16522 & 600 & 63 & 13 \\
\hline 53003.41914 & 600 & -20 & 17 & 53008.17352 & 600 & 43 & 13 \\
\hline 53003.42765 & 600 & 5 & 19 & 53008.18212 & 600 & 78 & 14 \\
\hline 53003.43626 & 600 & 9 & 21 & 53008.19032 & 600 & 66 & 15 \\
\hline 53003.47535 & 1800 & 60 & 28 & 53008.19842 & 600 & 71 & 14 \\
\hline 53004.12513 & 600 & 48 & 13 & 53008.20713 & 600 & 39 & 12 \\
\hline 53004.13348 & 600 & 20 & 16 & 53008.21590 & 600 & 39 & 12 \\
\hline 53004.14188 & 600 & 61 & 13 & 53008.22407 & 600 & 48 & 13 \\
\hline 53004.15034 & 600 & 60 & 18 & & & & \\
\hline
\end{tabular}




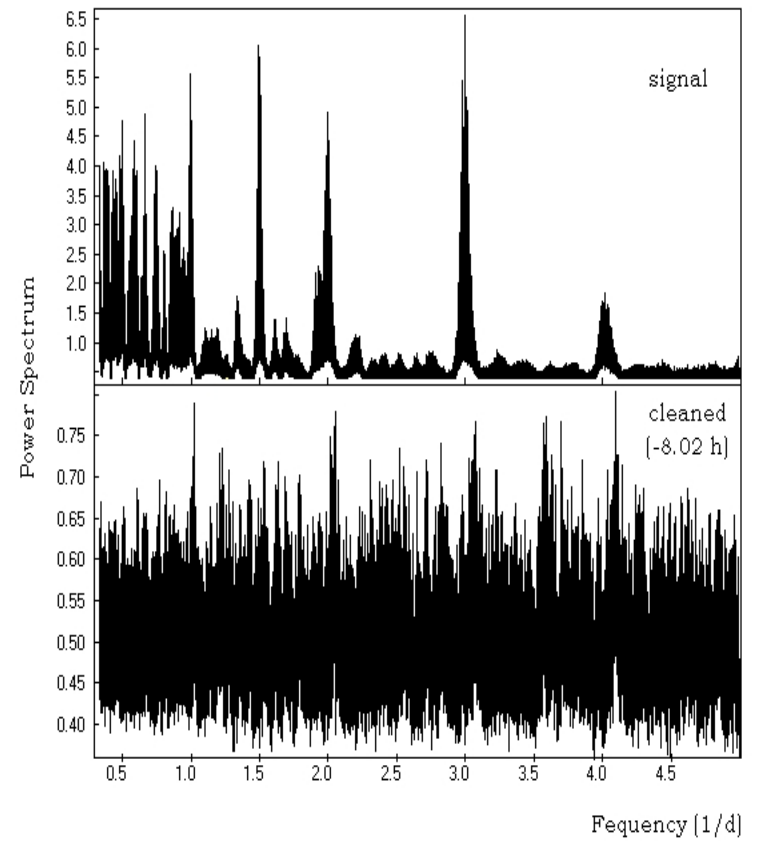

Fig. 1. Power spectrum the magnetic field variations of WD 0009+501 (upper plot) and cleaned spectrum (lower plot) for the main sinusoidal signal $P \approx 8 \mathrm{~h}$.

Finally, our previous observations (Fabrika et al. 2003), together with the data from Schmidt \& Smith (1994) made it possible to further test the realibity of our estimate of the rotation period. A combined analysis of the measurements with the data of Fabrika et al. (2003) and Schmidt \& Smith (1994) confirmed that the best fit is obtained adopting a rotation period of about $8 \mathrm{~h}$. The magnetic phase curve of WD 0009+501 derived with this period is presented in Fig. 2. The phase curve is almost sinusoidal and symmetrical about the value of about $-30 \mathrm{kG}$. The longitudinal magnetic field varies from $-120 \pm 10 \mathrm{kG}$ to $+50 \pm 5 \mathrm{kG}$.

The period derived is in good agreement with all other available observations. For the maximum of the mean longitudinal field we calculate the following ephemeris:

$\mathrm{JD}=2453003.34 \pm 0.005+0.334 \pm 0.008 E$.

\section{Phase resolved $I$ and $V$ Stokes spectra. Determinations of mean longitudinal field, mean field modulus, and crossover}

After the determination of the rotation period via analysis of the $B_{1}$ measurements, we combined spectra using four consecutive exposures obtained at two orthogonal orientations of the quarter-wave plate (the sequence of its position angles is $+45^{\circ},-45^{\circ},-45^{\circ},+45^{\circ}$ ). The resulting combined exposures correspond to a total of $600 \mathrm{~s}$ integration time as logged in Table 1. From these spectra, we obtained individual Stokes $I$ and $V$ by applying the technique presented by Naydenov et al. (2002; see also Aznar Cuadrado et al. 2004). Finally, we build phase-resolved Stokes $I$ and $V$ spectra by further combining individual Stokes $I$ and $V$ spectra into 20 phase bins

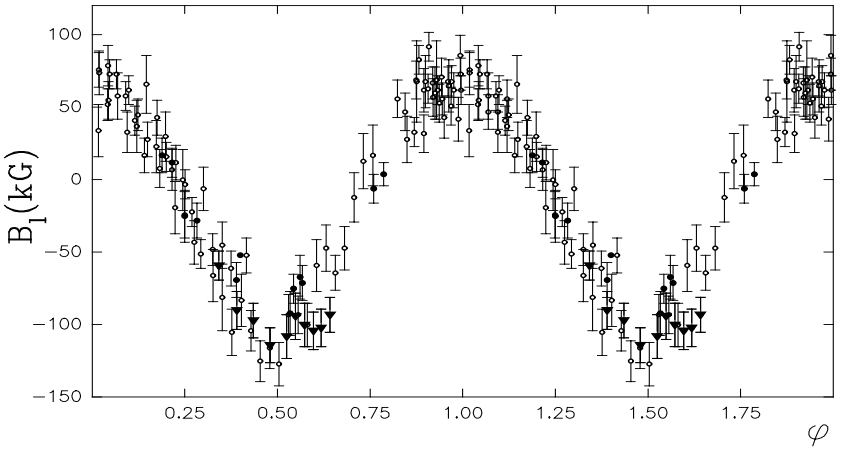

Fig. 2. The magnetic phase curve of WD $0009+501$ with the 8-h period. Empty circles indicate the new observations presented in this paper, filled circles are observations by Schmidt \& Smith (1994) and triangles are the data by Fabrika et al. (2003).
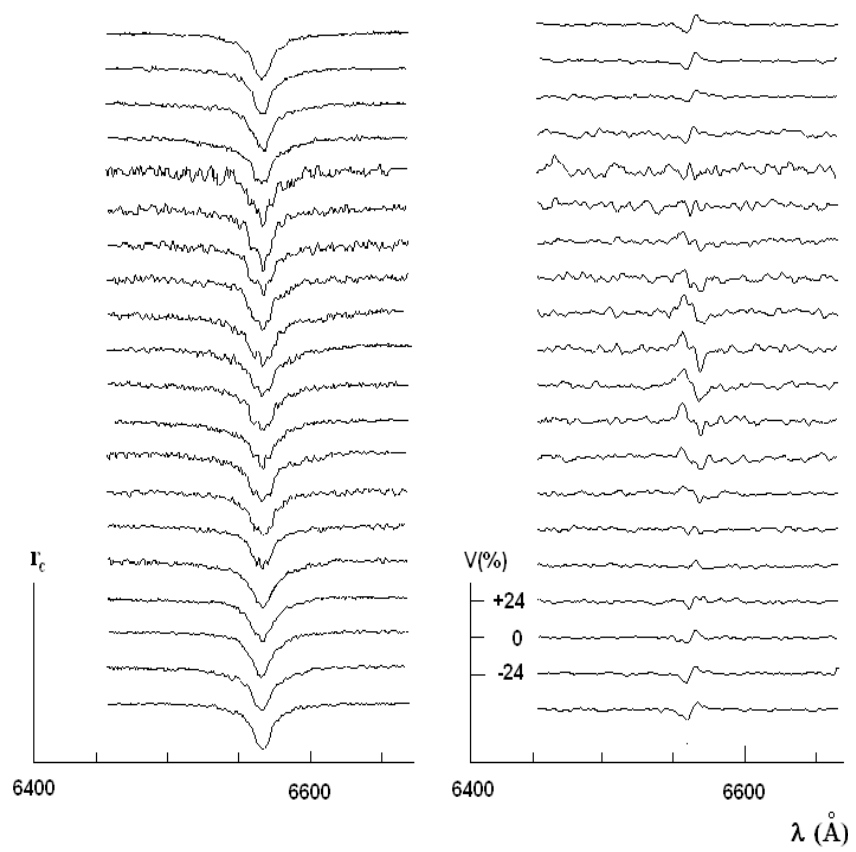

Fig. 3. Phase-resolved Stokes $I$ (left) and Stokes $V$ (right) profiles of WD $0009+501$ in the $\mathrm{H} \alpha$ region phased with the period $P=8 \mathrm{~h}$ and binned in $0.05 \phi$ intervals. The rotation phase increases from bottom to top, $\phi=0$ corresponds to the $B_{1}$ maximum.

taken 0.05 apart. In all cases, the coadditions of individual Stokes $I$ and $V$ profiles was performed weigthing the individual spectra with coefficients that depend on the noise.

The final Stokes $I$ and $V$ profiles are presented in Fig. 3. The rotation phase in the figure increases from bottom to top, $\phi=0$ corresponds to maximum $B_{1}$. The magnetic variablility of the $\mathrm{H} \alpha$ Stokes $V$ profile is clearly seen.

Figure 4 shows the $\mathrm{H} \alpha$ profile in three specific phases: $\phi=0$ (the maximum field $B_{1} \approx 50 \mathrm{kG}$ ), the crossover (zero $B_{1}$ ) and $\phi=0.5$ (the minimum field $B_{1} \approx-120 \mathrm{kG}$ ). The resolved Zeeman triplet at the moment of the minimum field provides the most reliable estimate of the surface magnetic field at this point: about $300 \mathrm{kG}$.

An initial inspection of the data did not reveal any evidence of important non-dipolar field components requiring a more 

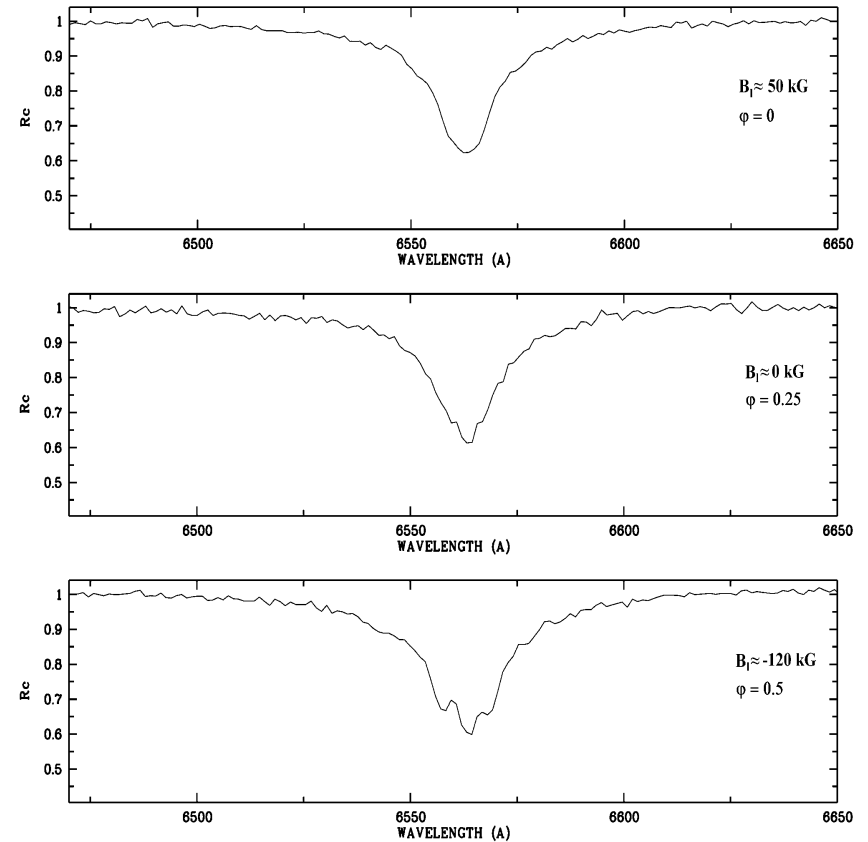

Fig. 4. H $\alpha$ line profiles of WD $0009+501$ (from top to bottom) in the maximum magnetic field $\left(B_{1} \approx 50 \mathrm{kG}\right)$, the crossover and in the minimum field $\left(B_{1} \approx-120 \mathrm{kG}\right)$.

sophisticated analysis using Zeeman tomography (e.g. Euchner et al. 2002). The Stokes $V$ spectra show a single S-shaped profile which changes sign during the stellar rotational cycle, indicative of a dominant dipolar component in the field. For this reason we do not model the detailed Stokes $V$ profiles, but consider the data in terms of mean observables obtained from the spectra: mean longitudinal field, surface field and crossover. Despite the fact that individual measurements of magnetic observables are only weakly sensitive to higher-order field components, these components can be detected by analysing the phase variation of the observables (Bagnulo et al. 2002). In order to perform a more detailed analysis of the Stokes $V$ profiles, higher signal-to-noise ratios are required.

\subsection{Mean longitudinal field determinations}

From the Stokes $I$ and $V$ profiles, binned in 0.05-cycle intervals, we have obtained the longitudinal field determinations through the expression

$V(\lambda) \sim B_{1}\left(\frac{\lambda}{\lambda_{0}}\right)^{2} \frac{1}{I_{\lambda}} \frac{\mathrm{d} I_{\lambda}}{\mathrm{d} \lambda}$,

where $\frac{\mathrm{d} I_{\lambda}}{\mathrm{d} \lambda}$ describes the flux profile, $\lambda_{0}$ is a Balmer line rest wavelength (see Angel et al. 1973; also Schmidt \& Smith 1995, and references therein).

The measurements are presented in Cols. 2 and 3 of Table 2, and plotted in the upper panel of Fig. 5. The longitudinal magnetic field shows almost sinusoidal variability with minor (but statistically significant) deviations. Using a first order Fourier expansion, $B_{1}=A+B \sin \left(2 \pi t / P+\phi_{0}\right)$ the best-fit was obtained with the parameters $A=-23 \pm 2 \mathrm{kG}, B=77 \pm 3 \mathrm{kG}$ (see thin solid lines in the top panel of Fig. 5).
Table 2. WD 0009+501: phase-resolved observable magnetic quantities longitudinal field, mean field modulus or "surface field", and crossover.

\begin{tabular}{lcccccc}
\hline \hline $\begin{array}{l}\text { Rot. } \\
\text { phase }\end{array}$ & $\begin{array}{c}B_{1} \\
\mathrm{kG})\end{array}$ & $\begin{array}{c}\sigma\left(B_{1}\right) \\
(\mathrm{kG})\end{array}$ & $\begin{array}{c}B_{\mathrm{s}} \\
(\mathrm{kG})\end{array}$ & $\begin{array}{c}\sigma\left(B_{\mathrm{s}}\right) \\
(\mathrm{kG})\end{array}$ & $\begin{array}{c}v_{\mathrm{e}} \sin i\left\langle\mathrm{~d} B_{z}\right\rangle \\
\left(\mathrm{kG} \mathrm{km} \mathrm{s}^{-1}\right)\end{array}$ & $\begin{array}{c}\left(v_{\mathrm{e}} \sin i\left\langle\mathrm{~d} B_{z}\right\rangle\right) \\
\left(\mathrm{kG} \mathrm{km} \mathrm{s}^{-1}\right)\end{array}$ \\
\hline 0.025 & +49 & 6 & 190 & 16 & -90 & 80 \\
0.075 & +42 & 5 & 163 & 18 & -69 & 70 \\
0.125 & +25 & 7 & 169 & 15 & +58 & 70 \\
0.175 & +17 & 9 & 155 & 15 & +250 & 65 \\
0.225 & -4 & 8 & 138 & 13 & +32 & 100 \\
0.275 & -11 & 7 & 187 & 14 & +25 & 130 \\
0.325 & -52 & 9 & 200 & 19 & -78 & 150 \\
0.375 & -73 & 14 & 228 & 18 & -360 & 230 \\
0.425 & -99 & 15 & 254 & 20 & +540 & 260 \\
0.475 & -119 & 14 & 259 & 23 & +13 & 235 \\
0.525 & -125 & 15 & 258 & 18 & -178 & 190 \\
0.575 & -105 & 14 & 288 & 22 & -380 & 220 \\
0.625 & -71 & 14 & 255 & 20 & -641 & 260 \\
0.675 & -63 & 9 & 300 & 30 & -425 & 320 \\
0.725 & -18 & 14 & 245 & 50 & +47 & 300 \\
0.775 & 0 & 16 & 250 & 75 & -208 & 580 \\
0.825 & +35 & 11 & 205 & 35 & -182 & 80 \\
0.875 & +42 & 6 & 191 & 17 & +100 & 90 \\
0.925 & +48 & 5 & 200 & 15 & -47 & 65 \\
0.975 & +53 & 4 & 181 & 15 & +168 & 70 \\
\hline & & & & & &
\end{tabular}

\subsection{Mean field modulus determinations}

The spectral resolution $(R \sim 2000)$ also makes it possible to estimate a surface magnetic field $B_{\mathrm{s}}$ at each phase bin. (The surface field is the modulus $|B|$ of the total magnetic vector integrated over the star's visible hemisphere.) To measure $B_{\mathrm{s}}$ we used estimative method based on deblending of the line core by means of simultaneous fit of three Gaussian profiles. Such a method makes it possible to reconstruct Zeeman pattern at each point of the phase curve and estimate the surface field by measurements of the displacement of the Gaussians. The measurements are presented in Cols. 4 and 5 of Table 2, and plotted in the middle panel of Fig. 5.

\subsection{The second-order moment of the Ho Stokes $V$ profile: crossover determinations}

We have attempted to measure the second order moment in phase-resolved Stokes $V$ spectra of the $\mathrm{H} \alpha$ profile, namely the quantity

$R_{V}^{(2)}=\frac{1}{W(\mathrm{H} \alpha)} \int V(\lambda)\left(\lambda-\lambda_{0}\right)^{2} \mathrm{~d} \lambda$

Mathys (1995) has shown that $R_{V}^{(2)}$ is an estimate of the so called mean asymmetry of the longitudinal field multiplied by $v_{\mathrm{e}} \sin i$, the crossover $v_{\mathrm{e}} \sin i\left\langle\mathrm{~d} B_{z}\right\rangle$. Unfortunately, our observations are characterized by a $S / N$ ratio that is too low to determine the crossover with sufficiently high precision. Integration 


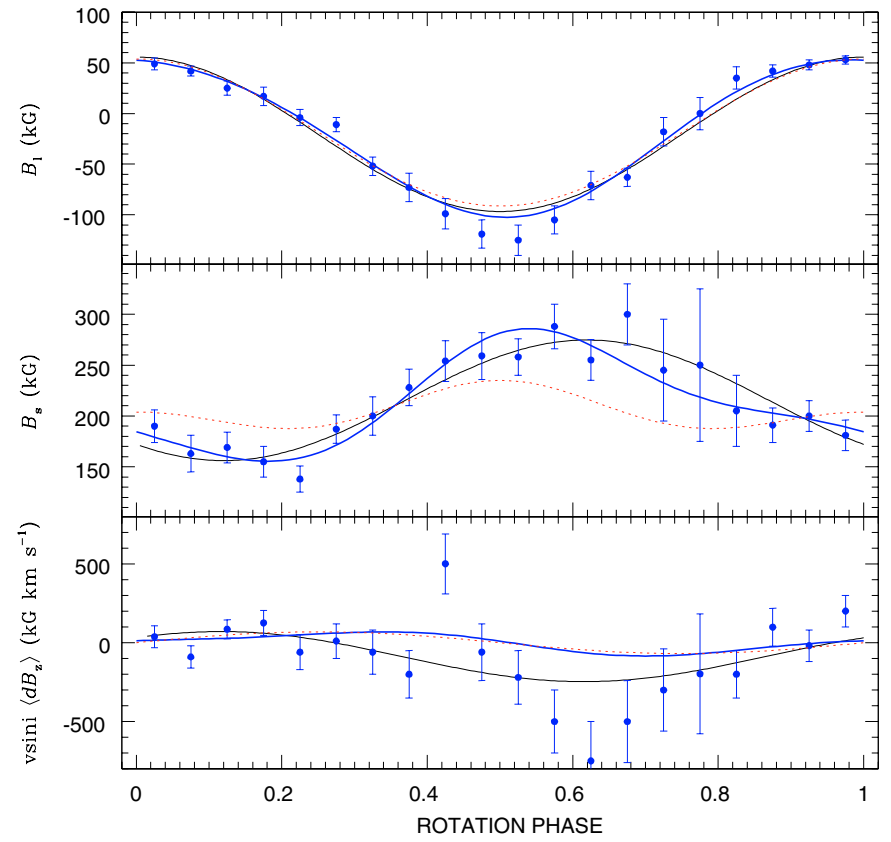

Fig. 5. Observations and modelling of mean longitudinal field (top panel), mean field modulus, or surface field (middle panel) and crossover (bottom panel). The thin solid lines show a fit obtained with simple second order Fourier expansion. Thick solid lines show the best-fit obtained by means of a dipole + quadrupole model. Dotted lines show the best-fit obtained assuming that a simple dipole model.

of Stokes $V$ in the wings of the $\mathrm{H} \alpha$ profile leads to very large error bars of $R_{V}^{(2)}$ because the noise grows as a quadratic function of the distance from $\lambda_{0}$ (the larger the spectral line width, the larger the impact of the error on Stokes $V$ to the final error bar on $\left.R^{(2)}\right)$. We therefore decide to integrate $V(\lambda)\left(\lambda-\lambda_{0}\right)^{2}$ only in central $\mathrm{H} \alpha$ regions where the Stokes $V$ parameter is significantly different from zero (typically $\pm 30 \AA$ from the $\mathrm{H} \alpha$ core). Such a way one may affect the result toward over- or underestimation of the second order moment. Nevertheless, we find it helpful to present the data here and use them in our consideration as a first illustration for the magnetic white dwarfs. The measurements are presented in Cols. 6 and 7 of Table 2, and plotted in the bottom panel of Fig. 5 .

\section{Modelling the magnetic field of WD $0009+501$}

It is generally assumed that the magnetic morphology of white dwarfs is organized at a large scale, and that can be possibly described by means of a low-order multipolar expansion (Putney 1999). To quickly see if the observations of WD $0009+501$ are consistent with a simple dipolar geometry, we have followed the schematic method proposed by Landolfi et al. (1997). Inspection of Fig. 5 shows that i) the mean field modulus curve has only two extrema (case I A of Eq. (6) of Landolfi et al. 1997); ii) the mean field modulus at phase 0 is smaller than the mean field modulus at phase 0.5, (case II B of Eq. (8) of Landolfi et al. 1997). Finally,

iii) $B_{1}^{\max }>0>B_{1}^{\min },\left|B_{1}^{\max }\right|<\left|B_{1}^{\min }\right|$,

(case 4 III of Eq. (4) of Landolfi et al. 1997). A comparison with Table 1 of Landolfi et al. (1997) shows that these features, all together, are not consistent with a pure dipolar configuration. Therefore we have decided to model the measurements of mean longitudinal field, mean field modulus, and crossover of Table 2 within the framework of a dipole plus quadrupole field as defined in Landolfi et al. (1998). The modelling technique is described in detail in Bagnulo et al. (2000). Here we recall that the curves of mean longitudinal field, surface magnetic field, and crossover, are interpreted in terms of a dipole plus quadrupole model. The best-fit to the observations is obtained through a Marquardt method of $\chi^{2}$ minimisation (Bevington 1969). The dipole plus quadrupole model depends on the following 10 parameters:

- $B_{\mathrm{d}}$ and $B_{\mathrm{q}}$, the dipole and quadrupole strength, respectively;

- $v_{\mathrm{e}}$, the stellar equatorial velocity;

- $i$, the inclination of the stellar rotation axis to the line of sight;

$-\beta$, the angle between the dipolar axis and the rotation axis;

$-\beta_{1}$ and $\beta_{2}$, the analogues of $\beta$ for the directions identified by the quadrupole;

$-\gamma_{1}$ and $\gamma_{2}$, the azimuthal angles of the unit vectors of the quadrupole;

- $f_{0}$, the "reference" rotational phase of the model.

The angles $i, \beta, \beta_{1}, \beta_{2}$ range from $0^{\circ}$ to $180^{\circ}$, while $\gamma_{1}, \gamma_{2}$, $f_{0}$ range from $0^{\circ}$ to $360^{\circ}$. The rotational period $P$ and the limbdarkening constant $u$, which also affects the expressions of the magnetic observables, are taken as fixed. (Note that the pure dipole model would retain as free parameters only $B_{\mathrm{d}}, v_{\mathrm{e}}, i, \beta$, and $f_{0}$.)

For the stellar mass, Wickramasinghe \& Ferrario (2000) estimated the value of $0.89 M_{\odot}$, which approximately corresponds to a stellar radius of $0.009 R_{\odot}$ (Hamada \& Salpeter 1961). Finally, for this work, we decided to set as a constraint $R / R_{\odot}=0.01 \pm 0.002$.

Based on our low resolution spectra, we are not able to set a strong constraint on $v_{\mathrm{e}} \sin i$. However, introducing our radius and period estimate in the formula

$v_{\mathrm{e}} \sin i \leq \frac{50.8 R / R_{\odot}}{P(\mathrm{~d})} \mathrm{km} \mathrm{s}^{-1}$,

we obtain that $v_{\mathrm{e}} \sin i$ must be less than $2 \mathrm{~km} \mathrm{~s}^{-1}$.

For the limb-darkening coefficient, we adopted the value of $u=0.5$. Note that, as discussed by Bagnulo et al. (2000), the results of the modelling are only slightly influenced by the $u$ value. The best-fit parameters are:

\begin{tabular}{|llrll|}
\hline$i$ & $=$ & $60^{\circ}$ & \pm & $20^{\circ}$ \\
$\beta$ & $=$ & $111^{\circ}$ & \pm & $17^{\circ}$ \\
$f_{0}$ & $=$ & $0^{\circ}$ & \pm & $3^{\circ}$ \\
$\beta_{1}$ & $=$ & $52^{\circ}$ & \pm & $35^{\circ}$ \\
$\beta_{2}$ & $=$ & $104^{\circ}$ & \pm & $27^{\circ}$ \\
$\gamma_{1}$ & $=$ & $331^{\circ}$ & \pm & $37^{\circ}$ \\
$\gamma_{2}$ & $=$ & $178^{\circ}$ & \pm & $40^{\circ}$ \\
$B_{\mathrm{d}}$ & $=$ & 338 & \pm & $70 \mathrm{kG}$ \\
$B_{\mathrm{q}}$ & $=$ & 278 & \pm & $208 \mathrm{kG}$ \\
$v_{\mathrm{e}}$ & $=$ & 1.53 & \pm & $0.15 \mathrm{~km} \mathrm{~s}^{-1}$ \\
\hline
\end{tabular}

and the assocated $v_{\mathrm{e}} \sin i$ and radius values are $1.32 \pm$ $0.40 \mathrm{~km} \mathrm{~s}^{-1}$ and $0.010 \pm 0.001 R_{\odot}$, respectively. 
Note that, as explained by Bagnulo et al. (2000), the available observations do not allow one to distinguish between two magnetic configurations symmetrical about the plane containing the rotation axis and the dipole axis. Such configurations are characterised by the same values of $B_{\mathrm{d}}, B_{\mathrm{q}}, v_{\mathrm{e}}, \gamma_{1}, \gamma_{2}, f_{0}$, while the remaining angles are related by

$$
\begin{array}{cccc}
(i, & \beta, & \beta_{1}, & \left.\beta_{2}\right) \\
\left(180^{\circ}-i,\right. & 180^{\circ}-\beta, & 180^{\circ}-\beta_{1}, & \left.180^{\circ}-\beta_{2}\right) .
\end{array}
$$

The best-fit to the observations is shown with solid lines in Fig. 5. For comparison, the dotted line shows the best-fit obtained using a simple dipolar morphology. The parameters of the best dipole model are

\begin{tabular}{|llrll|}
\hline$i$ & $=$ & $63^{\circ}$ & \pm & $24^{\circ}$ \\
$\beta$ & $=$ & $117^{\circ}$ & \pm & $24^{\circ}$ \\
$f_{0}$ & $=$ & $1^{\circ}$ & \pm & $2^{\circ}$ \\
$B_{\mathrm{d}}$ & $=$ & 295 & \pm & $6 \mathrm{kG}$ \\
$v_{\mathrm{e}}$ & $=$ & 1.50 & \pm & $0.15 \mathrm{~km} \mathrm{~s}^{-1}$ \\
\hline
\end{tabular}

and the associated $v_{\mathrm{e}} \sin i$ and stellar radius values are $1.34 \pm$ $0.25 \mathrm{~km} \mathrm{~s}^{-1}$ and $0.010 \pm 0.001 R_{\odot}$, respectively. It is worthwhile to note that the dipole parameters are very similar in both models, whereas the quadrupolar component of the dipole+quadrupole model is left quite undetermined due to the large error bars.

The detection of a significant quadrupolar component is not a big surprise. Returning back to our previous observations of WD $0009+501$ (Fabrika et al. 2003) we note that the (wrong) estimate of $2 \mathrm{~h}$ for the rotational period was based on the analysis of observations obtained in a 2.5 interval, during which the star showed negative longitudinal field. In Fabrika et al. (2003), our observations of a noise bimodal structure around the negative extremum of the field had been erronesouly interpreted as the modulation due to a full rotation cycle. In fact, the observed bimodal structure (that is washed out in the rebinning of the data) could possibly be the signature of field structure even more complex than a dipole plus quadrupole.

\section{Conclusions}

We have presented new observations of low-resolution Stokes $I$ and $V$ profiles of the magnetic white dwarf WD $0009+501$. From these and previous observations we have determined the star's rotation period, we have determined the mean longitudinal field, the mean field modulus, and the crossover. Finally we have developed a magnetic model consistent with all the observations.

The rotation period of WD $0009+501$ is $0.334 \pm 0.008 \mathrm{~d}$, based on our new observations as well as archival data obtained by Fabrika et al. (2003) and Schmidt \& Smith (1994).

The longitudinal magnetic field of WD 0009+501 varies from $-120 \pm 10 \mathrm{kG}$ to $+50 \pm 5 \mathrm{kG}$. The surface field shows a variation between $\approx 150$ and $\approx 300 \mathrm{kG}$.

Assuming the magnetic field of WD $0009+501$ to be a dipole we found its basic parameters: the rotation axis inclination is about $60^{\circ}$ and the dipole axis inclination about $120^{\circ}$; the field strength at the pole, $B_{\mathrm{d}}$, is about $295 \mathrm{kG}$. However, it is clear from the fit to the observations that the dipole model is not sufficient to explain all available observations.

Assuming the magnetic field of WD 0009+501 is given by the superposition of a dipole and quadrupole, we found that the rotation axis inclination is about $60^{\circ}$ and the dipole axis inclination about $110^{\circ}$; the polar field of the dipole $B_{\mathrm{d}} \approx 340 \mathrm{kG}$; the quadrupole field $B_{\mathrm{q}}$ of about $300 \mathrm{kG}$. The orientation of the dipolar angles are almost the same as obtained under the assumption of a pure dipolar model, but the quadrupole configuration is left quite undeterminated because of the large error bars associated to the model parameters.

Landstreet \& Mathys (2000) have discovered that slow rotating magnetic Ap stars (i.e. with rotation period of about 1 month or longer) have a magnetic axis tilted at a small angle with respect to the rotation axis. Landstreet \& Mathys (2000) and Bagnulo et al. (2002) suggest also that faster rotating stars tend to have a magnetic axis that forms a large angle with the rotation axis. Taking into account fairly short rotation period of the degenerate we may hypothesize that white dwarf magnetic fields display similar correlations with rotational period as those of the magnetic Ap/Bp stars. According to Landstreet \& Mathys (2000), long-period magnetic Ap/Bp stars exhibit small magnetic tilt angles (i.e. magnetic axes nearly aligned with rotational axes), whereas short-period $\mathrm{Ap} / \mathrm{Bp}$ stars exhibit large tilt angles (i.e. magnetic axes nearly perpendicular to rotational axes). In WD0009+501, we have found that the dipolar axis of the field forms a large angle with respect to the rotational axis. It would be interesting to explore whether the field tilt angle is also correlated with rotational period in the MWDs. If established on a sound statistical basis, this result could imply that there are universal dynamical mechanisms in the magnetic main sequence stars and their remnants worthy of additional study.

Finally, it should be noted that the polar strength of the dipole of WD $0009+501$ is about $300 \mathrm{kG}$. Assuming magnetic flux conservation during MS star evolution into a white dwarf, we estimate that the dipole strength during the star's life on the main sequence was of a few tens of Gauss. This implies that either Ap stars with extremely weak dipole fields exist (which is not supported by the results of Auriere et al. 2004, who find that all Ap stars have dipole fields stronger than about $300 \mathrm{G}$ ), or that a significant flux loss occurs during the post-main sequence evolutionary processes, or that stars other than magnetic chemically peculiar Ap/Bp stars evolve into magnetic white dwarfs. The last assumtion has been recently supported by Wickramasinghe \& Ferrario (2005). They argue that magnetic white dwarfs have progenitors that are not restricted to $\mathrm{Ap} / \mathrm{Bp}$ magnetic stars. To address these issues, further studies of weak-field white dwarfs are needed.

Acknowledgements. G.V. and G.G. are grateful to the Korean MOST (Ministry of Science and Technology, grant M1-022-00-0005) and KOFST (Korean Federation of Science and Technology Societies) for providing them an opportunity to work at KAO through Brain Pool program. G.V. and S.F. acknowledge the Russian Foundation for Basic Research for financial support (RFBR grant No. 01-0216808). G.A.W. acknowledges Discovery Grant support from the Natural Sciences and Engineering Research Council of Canada. G.G. also wants to express his thanks to the Russian Foundation for Basic 
Research (under the grant No. 02-02-17423) and the Federal program "Astronomy" for financial support.

The authors are especially grateful to Prof. John Lanstreet and anonymous referee for their valuable communications and comments on the manuscript.

\section{References}

Afanasiev, V. L., Burenkov, A. N., Vlasyuk, V. V., \& Drabek, S. V. 1995, SAO Ras internal Rep., 234

Angel, J. R. P., McGraw, J. T., \& Stockman, H. S. 1973, ApJ, 184, L79

Angel, J. R. P., Borra, E. F., \& Landstreet, J. D. 1981, ApJS, 45, 457

Aznar Cuadrado, R., Jordan, S., Napiwotzki, R., et al. 2004, A\&A, 423, 1081

Auriere, M., Donati, J.-F. F., Lignieres, D., et al. 2004, in The A-Star Puzzle, the Proc. IAU Symp., 224, in press

Bagnulo, S., Landolfi, M., Mathys, G., \& Landi Degl'Innocenti, M. 2000, A\&A, 358, 929

Bagnulo, S., Landi Degl'Innocenti, M., \& Landolfi, M. 2002, A\&A, 394, 1023

Bevington, P. R. 1969, Data reduction and error analysis for the physical sciences (New York: McGraw-Hill)

Euchner, F., Jordan, S., Beuermann, K., Gänsicke, B. T., \& Hessman, F. V. 2002, A\&A, 390, 633

Fabrika, S. N., Valyavin, G. G., \& Burlakova, T. E. 2003, Astron. Lett., 29,737

Goransky, V. 2004, private communication

Hamada, T., \& Salpeter, E. E. 1961, ApJ, 134, 683

Klochkova, V. G., Yermakov, S. V., \& Panchuk, V. E. 1996, Bull. Spec. Astrophys. Obs., 41, 58
Lafler, J., \& Kinman, T. D. 1965, ApJS, 11, 216

Landolfi, M., Bagnulo, S., Landi Degl'Innocenti, E., Landi Degl'Innocenti, M., \& Leroy, J. L. 1997, A\&A, 322, 197

Landolfi, M., Bagnulo, S., \& Landi Degl'Innocenti, M. 1998, A\&A, 338,111

Landstreet, J. D., \& Mathys, G. 2000, A\&A, 359, 213

Liebert, J., Bergeron, P., \& Holberg, J. B. 2003, AJ, 125, 348

Mathys, G. 1995, A\&A, 293, 733

Maxted, P. F. L., Ferrario, L., Marsh, T. L., \& Wickramasinghe, D. T. 2000, MNRAS, 315, L41

Monin, D. N., Fabrika, S. N., \& Valyavin, G. G. 2002, A\&A, 396, 131

Muslimov, A. G., Van Horn, H. M., \& Wood, M. A. 1995, ApJ, 442, 758

Naydenov, I. D., Valyavin, G. G., Fabrika, S. N., et al. 2002, Bull. Spec. Astrophys. Obs., 53, 124

Putney, A. 1999, 11th European Workhop on White Dwarfs, ed. J. E. Solheim, \& E. G. Meistas, ASP Conf. Ser., 196, 195

Scargle, J. D. 1989, ApJ, 343, 874

Schmidt, G. D., \& Smith, P. S. 1994, ApJ, 423, L63

Schmidt, G. D., \& Smith, P. S. 1995, ApJ, 448, 305

Terebizh, V. 1992, Analysis of time rows in astrophysics, in Russian (Nauka: Moscow)

Valyavin, G. G., Burlakova, T. E., Fabrika, S. N., \& Monin, D. N. 2003, Astron. Rep., 47, 589

Wade, G. A., Bagnulo, S., Szeifert, T., et al. 2003, in Solar Polarization, ed. J. Trujillo Bueno, \& J. Sánchez Almeida, ASP Conf. Ser., 307, 565

Wendell, C. E., Van Horn, H. M., \& Sargent, D. 1987, ApJ, 313, 284

Wickramasinghe, D. T., \& Ferrario, L. 2000, PASP, 112, 873

Wickramasinghe, D. T., \& Ferrario, L. 2005, MNRAS, 356, 1576 